\title{
ON THE TOPOLOGICAL PROPERTIES OF THE CERTAIN NEURAL NETWORKS
}

\author{
Jia-Bao Liu ${ }^{1,2}$, Jing Zhao ${ }^{2}$, Shaohui Wang ${ }^{3}$, M. Javaid ${ }^{4}$, Jinde Cao ${ }^{1}$ \\ ${ }^{1}$ School of Mathematics, Southeast University, Nanjing, \\ Jiangsu, 210096, P.R. China \\ ${ }^{2}$ School of Mathematics and Physics, Anhui Jianzhu University, \\ Hefei, P.R. China \\ ${ }^{3}$ Department of Mathematics, Savannah State University, \\ Savannah, GA 31404, USA \\ ${ }^{4}$ Department of Mathematics, School of Science, University of \\ Management and Technology, Lahore, Pakistan
}

Submitted: 13th January 2018; Accepted: 08th March 2018

\begin{abstract}
A topological index is a numeric quantity associated with a network or a graph that characterizes its whole structural properties. In [Javaid and Cao, Neural Computing and Applications, DOI 10.1007/s00521-017-2972-1], the various degree-based topological indices for the probabilistic neural networks are studied. We extend this study by considering the calculations of the other topological indices, and derive the analytical closed formulas for these new topological indices of the probabilistic neural network. Moreover, a comparative study using computer-based graphs has been carried out first time to clarify the nature of the computed topological descriptors for the probabilistic neural networks. Our results extend some known conclusions.
\end{abstract}

Keywords: neural network, topological indices, Graph theory

\section{Introduction}

During the past decades, various neural networks have been undergoing for a rapid development in the various areas of studies, such as neurochemistry, artificial intelligence, automatic control and informational sciences [1]. The neural networks are used for the calcium oscillation behavior in biological mathematical models [2], especially in the powerful brain-like "neural" computers [3]. In the studies of quantitative structure activity relationships (QSAR models) and quantitative structure property relationships (QSPR models), the physicochemical properties and topological indices are excellent tools to explore the biological and chemical activities of the chemical compounds $[4,5]$.

The numerical quantities which transform networks/compounds structures to a numerical number are called topological indices/descriptors. More precisely, a topological index $\operatorname{Top}(G)$ is a function of a graph, in which if $H$ is isomorphic to $G$, then $\operatorname{Top}(H)=\operatorname{Top}(G)$. In general, there are three major classes of topological indices which are distancebased topological indices, degree-based topological indices and counting related indices of graphs [6]. Topological indices are the useful tools provided by 
networks theory for theoretical study of networks properties.

Since the Sierpinski networks are the first nontrivial families of graphs, which have been extensively studied in the last few years. M.Imran et al. [7] had obtained the formulas for the $A B C$ index, $G A$ index and fourth and fifth version of the $A B C$ and $G A$ index in the Sierpinski networks in 2017. The further results of topological indices of the generalized tree and $k$-tree [8] have been presented by S.H.Wang et al. For the probabilistic neural networks, M.Javaid et al. [9] have investigated the degree based topological indices of them in 2017. More studies about topological indices of other networks, one may refer to $[10,11]$.

Motivated by a large number of applications on topological indices for the networks theory, the information regarding topological indices are obtained from the probabilistic neural networks (PNN's) which consist of three types of layers. Assume that first, second and third layers called by input, hidden and output layers have $n$ nodes, $k$ classes (each class contains $m$ nodes) and $k$ nodes, respectively [9]. In the construction of PNN, each node of an input layer is connected to all the nodes of each class of the hidden layer and all the nodes of each class of the hidden layer are connected to the unique corresponding node of the output layer. In Fig. 1, a PNN denoted by $P N N(n, k, m)$ is shown for $n=4, k=2$ and $m=3$.

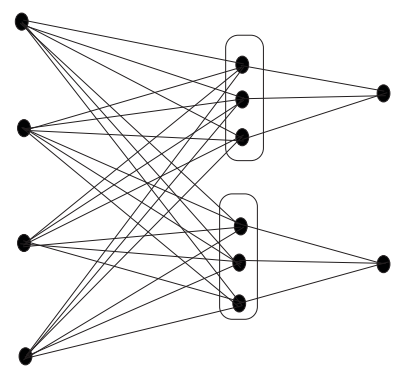

Figure 1. $P N N(4,2,3)$

The remaining of this paper is schematized as follows. Section 2 presents some preliminaries including the definitions and formulas. In Section 3, the main results are obtained for the TI's of the PNN and we conclude the whole paper in Section 4.

\section{Preliminaries}

A network is considered to be a connected graph having no multiple edges and no loops. We only consider finite and simple graphs here. Let $G=(V(G), E(G))$ be a graph with vertex-set $V(G)$ and edge-set $E(G)$. If $e \in E(G)$ has end vertices $u$ and $v$, then we say that $u$ and $v$ are adjacent and this edge is denoted by $u v$. The cardinality of $V(G)$ is called the order of $G$. Let $d_{i}=d\left(v_{i}\right)$ be the degree of vertex $v_{i}$ and $d(u, v)$ be the distance which is the length of the shortest path between $u$ and $v$. The notations used in this article are mainly taken from the book [12].

The Wiener index of a connected graph $G$ [13], denoted by $W(G)$, is recognized as the first proposed topological index, which is defined to be the sum of distances between every pair of vertices in $G$, i.e.,

$$
W(G)=\sum_{\{u, v\} \subseteq V(G)} d(u, v) .
$$

The Harary index of a connected graph $G$, denoted by $H(G)$, is defined as

$$
H(G)=\sum_{\{u, v\} \subseteq V(G)} \frac{1}{d(u, v)} .
$$

During the past decades, it has received much attention [14]. Nowadays, several variants of Harary index are introduced from the theoretical or applied viewpoint [15]. There are some varying Harary indices, a part of these are listed below.

$$
\begin{aligned}
H_{t}(G) & =\sum_{\{u, v\} \subseteq V(G)} \frac{1}{d(u, v)+t}, \\
H_{A}(G) & =\sum_{\{u, v\} \subseteq V(G)} \frac{d(u)+d(v)}{d(u, v)}, \\
H_{M}(G) & =\sum_{\{u, v\} \subseteq V(G)} \frac{d(u) \times d(v)}{d(u, v)} .
\end{aligned}
$$

Moreover, for the additively weighted Harary index, there is another name which is reciprocal degree distance. And the name is from the celebrated topological index in chemical graph theory [16], which is called $D D(G)$ [17]. Furthermore, the definition of $D D(G)$ is given by

$$
D D(G)=\sum_{\{u, v\} \subseteq V(G)}[d(u)+d(v)] d(u, v) .
$$


In the 1980s, Narumi and Katayama firstly considered the $N K(G)$ index [18], which is defined as

$$
N K(G)=\prod_{v \in V(G)} d(v) .
$$

In [19], Klein and Rosenfeld investigated some properties of this topological index. In 1988, H.Hosoya introduced Hosoya polynomial as a counting polynomial. Later on, Hosoya polynomial for a vertex of a graph was introduced by Gutman [20], which is associated with Hosoya polynomial of the graph. As we know, its definition is given by

$$
H(G, x)=\sum_{\{u, v\} \subseteq V(G)} x^{d(u, v)} .
$$

Among the most significant molecular descriptors, the classically molecular invariants are named as Zagreb indices, which are expressed as expected formulas for the total $\pi$-electron energy of conjugated molecules [21]. As the known results and wide applications about Zagreb indices, we discuss the varying Zagreb indices, and they are given by

$$
\begin{aligned}
& \overline{M_{1}}(G) \quad=\sum_{u \neq v, u v \notin E(G)}[d(u)+d(v)], \\
& \overline{M_{2}}(G) \quad=\sum_{u \neq v, u v \notin E(G)}[d(u) \times d(v)] .
\end{aligned}
$$

Let $f(u)$ be any function of vertex $u$, and it is obeyed the following guidelines

$$
\sum_{u \in V(G)} f(u)=\sum_{u v \in E(G)}\left[\frac{f(u)}{d((u)}+\frac{f(v)}{d(v)}\right] .
$$

It is worthy to mention that special case for $f(u)=$ $d(u)^{3}$ is known as $F-$ coindex [22]. In this paper, we will discuss a varying $F$-coindex, which is given by [23]

$$
\bar{F}(G)=\sum_{u v \notin E(G)}\left[d^{2}(u)+d^{2}(v)\right] .
$$

The irregularity index is the number of distinct terms of the degree sequence of $G$. It was introduced by S.Mukwembi [24] and its definition is given by

$$
\operatorname{irr}(G)=\sum_{u v \in E(G)}|d(u)-d(v)| .
$$

For the first multiplication Zagreb index in details, one may refer to $[25,26,27]$. Todeschini et al. proposed the addition of additive graph invariants in the Zagreb index, and its definition is given by

$$
\prod_{1}(G)=\prod_{i=1}^{n}\left[d\left(v_{i}\right)\right]^{2} .
$$

In 2000, Ivanciuc [28] and Ivanciuc et al. [29] firstly introduced the reciprocal complementary Wiener index, which is defined as

$$
R C W(G)=\sum_{\{u, v\} \subseteq V(G)} \frac{1}{d+1-d(u, v)},
$$

where $d$ is the diameter of $G$.

\section{Main Results}

This Section includes the main results of the present study.

Theorem 1 Let $G \cong P N N(n, k, m)$, where $n, k, m \geq$ 1. Then, the Wiener index of $G$ is given as

$W(G)=n(n-1)+k m(k m+n+3 k-3)+2 k(k+n-1)$.

Proof. According to the definition of $W(G)$ in Section 2, $V(G)$ have the following six cases for calculating the distances between any two distinct vertices in $\operatorname{PNN}(n, k, m)$.
(1) $u, v \in V_{1}$,
(2) $u, v \in V_{2}$,
(3) $u, v \in V_{3}$,
(4) $u \in V_{1}, v \in V_{2}$,
(5) $u \in V_{1}, v \in V_{3}$,
(6) $u \in V_{2}, v \in V_{3}$.

Based on the above cases, one can get

$$
\begin{aligned}
W(G)= & \sum_{u, v \in V_{1}} d(u, v)+\sum_{u, v \in V_{2}} d(u, v)+\sum_{u, v \in V_{3}} d(u, v) \\
& +\sum_{u \in V_{1}, v \in V_{2}} d(u, v)+\sum_{u \in V_{1}, v \in V_{3}} d(u, v) \\
& +\sum_{u \in V_{2}, v \in V_{3}} d(u, v) \\
& =2 C_{n}^{2}+2 C_{k m}^{2}+4 C_{k}^{2}+k m n+2 k n \\
& +k m+3 k m(k-1) \\
& =n(n-1)+k m(k m-1)+2 k(k-1)+k m n \\
& +2 k n+k m+3 k m(k-1) \\
& =n(n-1)+k m(k m+n+3 k-3) \\
& +2 k(k+n-1) .
\end{aligned}
$$


Theorem 2 Let $G \cong P N N(n, k, m)$, where $n, k, m \geq$ 1. Then, the Harary indices $H(G), H_{t}(G), H_{A}(G)$ and $H_{M}(G)$ are given as

$$
\begin{aligned}
& H(G)=\frac{n(n-1)}{4}+\frac{k m(k m-1)}{4}+\frac{k(k-1)}{8}+\frac{k n}{2}+ \\
& H_{A}(G)=\frac{k m n(n-1)}{2}+\frac{k m(k m-1)(n+1)}{2}+\frac{k m(k-1)}{4}+
\end{aligned}
$$

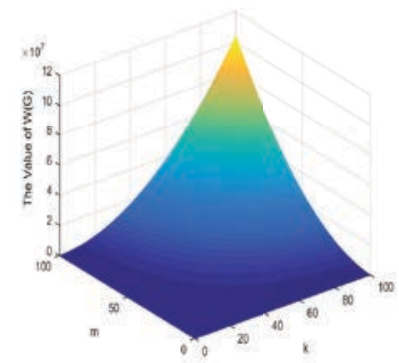

(a) $n=100$.

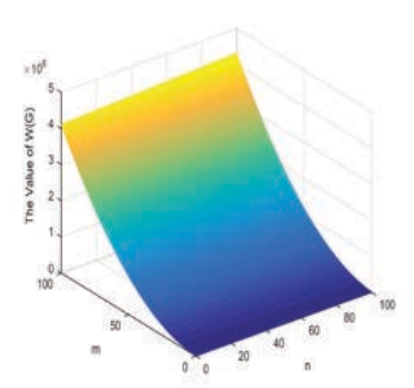

(b) $k=20$.

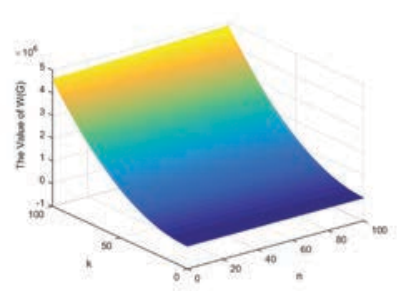

(c) $m=20$.

Figure 2. Computer-based comparative graph of the Wiener index for $\operatorname{PNN}(n, k, m)$ and its expression is $W(G)=$ $n(n-1)+k m(k m+n+3 k-3)+2 k(k+n-1)$.

Proof. According to the definition of Harary in- dex, the distances between any two distinct vertices in $\operatorname{PNN}(n, k, m)$ have the following six cases (1) $u, v \in V_{1}$, (2) $u, v \in V_{2}$, (3) $u, v \in V_{3}$, (4) $u \in$ $V_{1}, v \in V_{2},(5) u \in V_{1}, v \in V_{3},(6) u \in V_{2}, v \in V_{3}$.

Based on the above cases, we can obtain

$$
\begin{aligned}
H(G) & =\sum_{\{u, v\} \subseteq V(G)} \frac{1}{d(u, v)} \\
& =\sum_{u, v \in V_{1}} \frac{1}{d(u, v)} \\
& +\sum_{u, v \in V_{2}} \frac{1}{d(u, v)}+\sum_{u, v \in V_{3}} \frac{1}{d(u, v)} \\
& +\sum_{u \in V_{1}, v \in V_{2}} \frac{1}{d(u, v)} \\
& +\sum_{u \in V_{1}, v \in V_{3}} \frac{1}{d(u, v)}+\sum_{u \in V_{2}, v \in V_{3}} \frac{1}{d(u, v)} \\
& =\frac{1}{2} C_{n}^{2}+\frac{1}{2} C_{k m}^{2}+\frac{1}{4} C_{k}^{2} \\
& +k m n+\frac{1}{2} k n+k m+\frac{k m(k-1)}{3} \\
& =\frac{n(n-1)}{4}+\frac{k m(k m-1)}{4}+\frac{k(k-1)}{8} \\
+ & \frac{k n}{2}+\frac{k m(3 n+k+2)}{3} .
\end{aligned}
$$

Under the definition of a varying Harary index, by the comparable approach as used in the $H(G)$, we can obtain the result as follows

$$
H_{t}(G)=\sum_{\{u, v\} \subseteq V(G)} \frac{1}{d(u, v)+t}
$$

$=\sum_{u, v \in V_{1}} \frac{1}{d(u, v)+t}+\sum_{u, v \in V_{2}} \frac{1}{d(u, v)+t}+\sum_{u, v \in V_{3}} \frac{1}{d(u, v)+t}+$ $\sum_{u \in V_{1}, v \in V_{2}} \frac{1}{d(u, v)+t}+\sum_{u \in V_{1}, v \in V_{3}} \frac{1}{d(u, v)+t}+\sum_{u \in V_{2}, v \in V_{3}} \frac{1}{d(u, v)+t}$

$$
\begin{aligned}
& \quad=\frac{1}{2+t} C_{n}^{2}+\frac{1}{2+t} C_{k m}^{2}+\frac{1}{4+t} C_{k}^{2}+\frac{1}{1+t} k m n+\frac{1}{2+t} k n+ \\
& \frac{1}{1+t} k m+\frac{1}{3+t} k m(k-1) \\
& =\frac{n(n-1)}{4+2 t}+\frac{k m(k m-1)}{4+2 t}+\frac{k(k-1)}{8+2 t}+\frac{k m n}{1+t}+\frac{k n}{2+t}+ \\
& \frac{k m}{1+t}+\frac{k m(k-1)}{3+t} .
\end{aligned}
$$

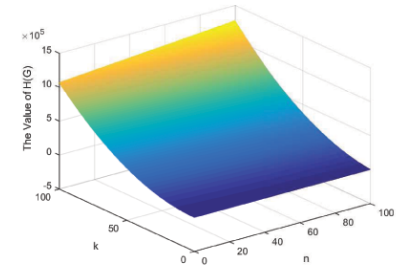

(a) $m=20$. 


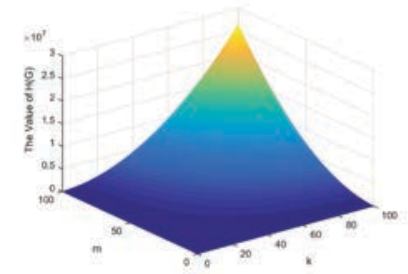

(b) $n=100$.

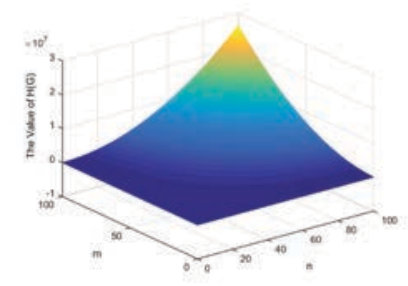

(c) $k=20$.

Figure 3. Computer-based comparative graph of the Harary index for $\operatorname{PNN}(n, k, m)$ and its expression is $H(G)=$ $\frac{n(n-1)}{4}+\frac{k m(k m-1)}{4}+\frac{k(k-1)}{8}+\frac{k n}{2}+\frac{k m(3 n+k+2)}{3}$.

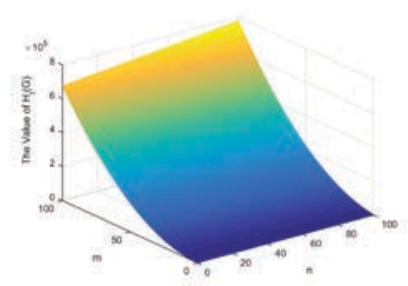

(a) $k=20, t=1$.

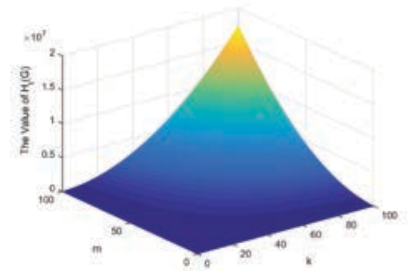

(b) $n=100, t=1$.

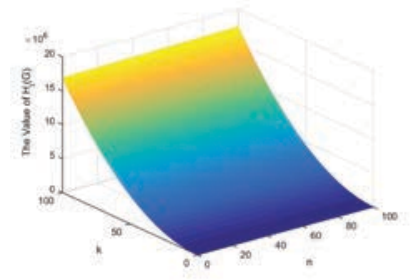

(c) $m=20, t=1$.

Figure 4. Computer-based comparative graph of the $H_{t}(G)$ index for $\operatorname{PNN}(n, k, m)$ and its expression is $H_{t}(G)=\frac{n(n-1)}{4+2 t}+\frac{k m(k m-1)}{4+2 t}+\frac{k n}{2+t}+$ $\frac{k(k-1)}{8+2 t)}+\frac{k m(n+1)}{1+t}+\frac{k m(k-1)}{3+t}$.
Especially, in the next two situations (based on the $H_{t}(G)$ ), let $t=1$ or 2 , respectively. Then we can obtain

$$
\begin{aligned}
H_{1}(G)= & \sum_{\{u, v\} \subseteq V(G)} \frac{1}{d(u, v)+1} \\
= & \sum_{u, v \in V_{1}} \frac{1}{d(u, v)+1}+\sum_{u, v \in V_{2}} \frac{1}{d(u, v)+1} \\
& +\sum_{u, v \in V_{3}} \frac{1}{d(u, v)+1} \\
& +\sum_{u \in V_{1}, v \in V_{2}} \frac{1}{d(u, v)+1} \\
& +\sum_{u \in V_{1}, v \in V_{3}} \frac{1}{d(u, v)+1}+\sum_{u \in V_{2}, v \in V_{3}} \frac{1}{d(u, v)+1} \\
= & \frac{1}{2+1} C_{n}^{2}+\frac{1}{2+1} C_{k m}^{2}+\frac{1}{4+1} C_{k}^{2} \\
& +\frac{1}{1+1} k m n+\frac{1}{2+1} k n \\
& +\frac{1}{1+1} k m+\frac{1}{3+1} k m(k-1) \\
= & \frac{n(n-1)}{6}+\frac{k m(k m-1)}{6}+\frac{k(k-1)}{10} \\
& +\frac{k m(n+1)}{2}+\frac{k n}{3}+\frac{k m(k-1)}{4} .
\end{aligned}
$$

And

$$
\begin{aligned}
H_{2}(G)= & \sum_{\{u, v\} \subseteq V(G)} \frac{1}{d(u, v)+2} \\
= & \sum_{u, v \in V_{1}} \frac{1}{d(u, v)+2}+\sum_{u, v \in V_{2}} \frac{1}{d(u, v)+2} \\
+ & \sum_{u, v \in V_{3}} \frac{1}{d(u, v)+2} \\
& +\sum_{u \in V_{1}, v \in V_{2}} \frac{1}{d(u, v)+2} \\
+ & \sum_{u \in V_{1}, v \in V_{3}} \frac{1}{d(u, v)+2}+\sum_{u \in V_{2}, v \in V_{3}} \frac{1}{d(u, v)+2} \\
= & \frac{1}{2+2} C_{n}^{2}+\frac{1}{2+2} C_{k m}^{2} \\
+ & \frac{1}{4+2} C_{k}^{2}+\frac{1}{1+2} k m n+\frac{1}{2+2} k n \\
& +\frac{1}{1+2} k m+\frac{1}{3+2} k m(k-1) \\
= & \frac{n(n-1)}{8}+\frac{k m(k m-1)}{8}+\frac{k(k-1)}{12} \\
+ & \frac{k m(n+1)}{3}+\frac{k n}{4}+\frac{k m(k-1)}{5} .
\end{aligned}
$$


Based on the definition of $H_{A}(G)=$ $\sum_{\{u, v\} \subseteq V(G)} \frac{d(u)+d(v)}{d(u, v)}$, the distances between any two distinct vertices in $\operatorname{PNN}(n, k, m)$ have the following six cases
(1) $u, v \in V_{1}$,
(2) $u, v \in V_{2}$,
(3) $u, v \in V_{3}$,
(4) $u \in V_{1}, v \in V_{2}$,
(5) $u \in V_{1}, v \in V_{3}$,
(6) $u \in V_{2}, v \in V_{3}$.

Based on the above cases, one can get

$$
\begin{aligned}
& H_{A}(G) \\
& =\sum_{u, v \in V_{1}} \frac{d(u)+d(v)}{d(u, v)}+\sum_{u, v \in V_{2}} \frac{d(u)+d(v)}{d(u, v)} \\
& +\sum_{u, v \in V_{3}} \frac{d(u)+d(v)}{d(u, v)}+\sum_{u \in V_{1}, v \in V_{2}} \frac{d(u)+d(v)}{d(u, v)} \\
& +\sum_{u \in V_{1}, v \in V_{3}} \frac{d(u)+d(v)}{d(u, v)}+\sum_{u \in V_{2}, v \in V_{3}} \frac{d(u)+d(v)}{d(u, v)} \\
& =\frac{2 k m}{2} C_{n}^{2}+\frac{2(n+1)}{2} C_{k m}^{2} \\
& +\frac{2 m}{4} C_{k}^{2}+k m n(k m+n+1)+\frac{k m+m}{2} k n \\
& +k m(1+m+n)+\frac{m+n+1}{3} k m(k-1) \\
& =\frac{k m n(n-1)}{2}+\frac{k m(k m-1)(n+1)}{2}+ \\
& \frac{k m(k-1)}{4}+k m n(k m+n+1) \\
& +\frac{k m n(k+1)}{2}+\frac{k m(m+n+1)(k+2)}{3} .
\end{aligned}
$$

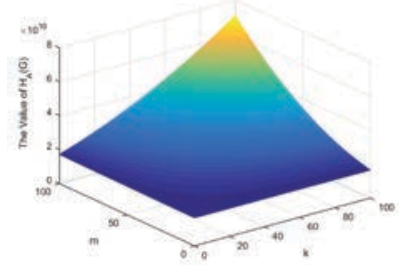

(a) $n=100$.

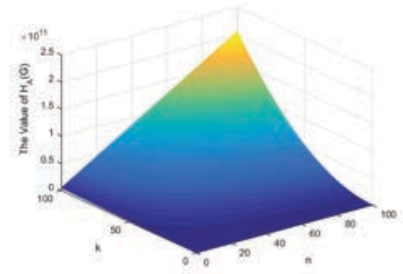

(b) $m=20$.

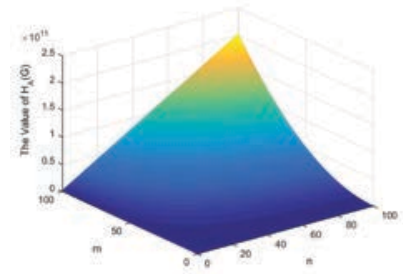

(c) $k=20$.

Figure 5. Computer-based comparative graph of the $H_{A}(G)$ index for $P N N(n, k, m)$ and its expression is

$H_{A}(G)=\frac{k m n(n-1)}{2}+\frac{k m(k m-1)(n+1)}{2}+\frac{k m(k-1)}{4}+$
$k m n(k m+n+1)+\frac{k m n(k+1)}{2}+\frac{k m(m+n+1)(k+2)}{3}$.

Similarly, according to the definition of $H_{M}(G)=\sum_{\{u, v\} \subseteq V(G)} \frac{d(u) \times d(v)}{d(u, v)}$, and its situations are same as $H_{A}(G)$, then we have

$$
\begin{aligned}
& H_{M}(G) \\
& =\sum_{u, v \in V_{1}} \frac{d(u) \times d(v)}{d(u, v)}+\sum_{u, v \in V_{2}} \frac{d(u) \times d(v)}{d(u, v)} \\
& +\sum_{u, v \in V_{3}} \frac{d(u) \times d(v)}{d(u, v)}+\sum_{u \in V_{1}, v \in V_{2}} \frac{d(u) \times d(v)}{d(u, v)} \\
& +\sum_{u \in V_{1}, v \in V_{3}} \frac{d(u) \times d(v)}{d(u, v)}+\sum_{u \in V_{2}, v \in V_{3}} \frac{d(u) \times d(v)}{d(u, v)} \\
& =\frac{k^{2} m^{2}}{2} C_{n}^{2}+\frac{(n+1)^{2}}{2} C_{k m}^{2}+\frac{m^{2}}{4} C_{k}^{2}+k m n[k m(n+1)]+ \\
& \frac{k m^{2}}{2} k n+k m[m(n+1)]+\frac{m(n+1)}{3} k m(k-1) \\
& =\frac{k^{2} m^{2} n(n-1)}{4}+\frac{k m(k m-1)(n+1)^{2}}{4}+\frac{k m^{2}(k-1)}{8} \\
& +\frac{k^{2} m^{2} n(2 n+3)}{2}+\frac{k m^{2}(n+1)(k+2)}{3} .
\end{aligned}
$$

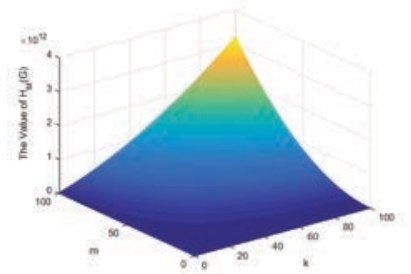

(a) $n=100$. 


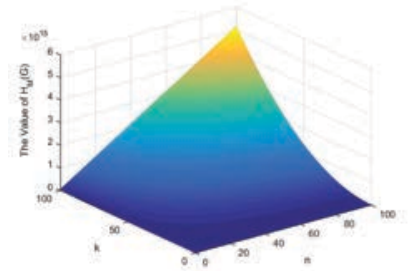

(b) $m=20$.

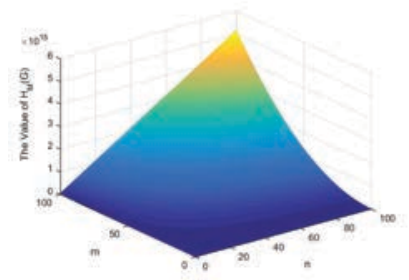

(c) $k=20$.

Figure 6. Computer-based comparative graph of the $H_{M}(G)$ index for $\operatorname{PNN}(n, k, m)$ and its expression is

$$
H_{M}(G)=\frac{k^{2} m^{2} n(n-1)}{4}+\frac{k m(k m-1)(n+1)^{2}}{4}+\frac{k m^{2}(k-1)}{8}+
$$

Theorem 3 Let $G \cong P N N(n, k, m)$, where $n, k, m \geq$ 1. Then the expression of $D D(G)$ is given as $D D(G)=k m n(k m+2 k+3 n+1)+2 k m(k m-$ 1) $(n+1)+4 k m(k-1)+k m(m+n+1)(3 k-2)$.

Proof. As a celebrated topological index in chemical graph theory, the distances between any two distinct vertices in $\operatorname{PNN}(n, k, m)$ have the following six cases
(1) $u, v \in V_{1}$,
(2) $u, v \in V_{2}$,
(3) $u, v \in V_{3}$,
(4) $u \in V_{1}, v \in V_{2}$,
(5) $u \in V_{1}, v \in V_{3}$,
(6) $u \in V_{2}, v \in V_{3}$.

Based on the definition of $D D(G)=$ $\sum_{\{u, v\} \subseteq V(G)}[d(u)+d(v)] d(u, v)$, we have

$D D(G)=\sum_{u, v \in V_{1}}[d(u)+d(v)] d(u, v)+$ $\sum_{u, v \in V_{2}}[d(u)+d(v)] d(u, v)+\sum_{u, v \in V_{3}}[d(u)+$ $d(v)] d(u, v)+\sum_{u \in V_{1}, v \in V_{2}}[d(u)+d(v)] d(u, v)+$ $\sum_{u \in V_{1}, v \in V_{3}}[d(u)+d(v)] d(u, v)+\sum_{u \in V_{2}, v \in V_{3}}[d(u)+$ $d(v)] d(u, v)$

$$
=4 k m C_{n}^{2}+4(n+1) C_{k m}^{2}+8 m C_{k}^{2}+k m n(k m+
$$
$n+1)+2 k m n(k+1)+k m(m+n+1)+3 k m(k-$ 1) $(m+n+1)$

$$
\begin{aligned}
& =k m n(3 n+k m+2 k+1)+2 k m(k m-1)(n+ \\
& 1)+4 k m(k-1)+k m(m+n+1)(3 k-2) .
\end{aligned}
$$

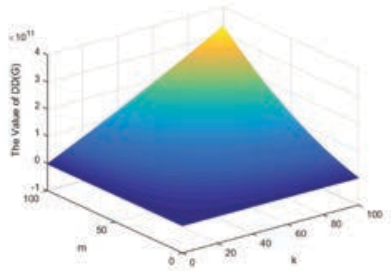

(a) $n=100$.

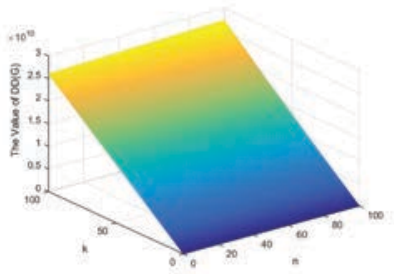

(b) $m=20$.

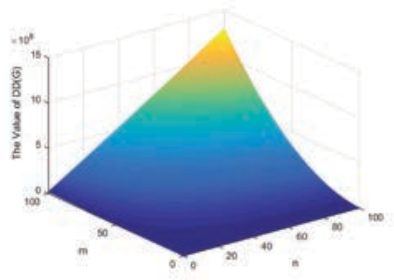

(c) $k=20$.

Figure 7. Computer-based comparative graph of the $D D(G)$ index for $P N N(n, k, m)$ and its expression is

$$
\begin{gathered}
D D(G)=k m n(k m+2 k+3 n+1)+2 k m(k m- \\
1)(n+1)+4 k m(k-1)+k m(m+n+1)(3 k-2) .
\end{gathered}
$$

Theorem 4 Let $G \cong P N N(n, k, m)$, where $n, k, m \geq$ 1. Then the expression of $H(G, x)$ is given as

$$
\begin{aligned}
& H(G, x)=\frac{k(k-1)}{2} x^{4}+k m(k-1) x^{3}+k n x^{2}+ \\
& \frac{n(n-1)}{2} x^{2}+\frac{k m(k m-1)}{2} x^{2}+k m x(n+1) .
\end{aligned}
$$

Proof. Obviously, the distances between any two distinct vertices in $\operatorname{PNN}(n, k, m)$ have the following six cases:
(1) $u, v \in V_{1}$,
(2) $u, v \in V_{2}$,
(3) $u, v \in V_{3}$,
(4) $u \in V_{1}, v \in V_{2}$,
(5) $u \in V_{1}, v \in V_{3}$,
(6) $u \in V_{2}, v \in V_{3}$.

Under the definition of $H(G, x)=\sum_{\{u, v\} \subseteq V(G)} x^{d(u, v)}$ and the above cases, one can get

$$
\begin{gathered}
H(G, x)=\sum_{u, v \in V_{1}} x^{d(u, v)}+\sum_{u, v \in V_{2}} x^{d(u, v)}+ \\
\sum_{u, v \in V_{3}} x^{d(u, v)}+\sum_{u \in V_{1}, v \in V_{2}} x^{d(u, v)}+\sum_{u \in V_{1}, v \in V_{3}} x^{d(u, v)}+ \\
\sum_{u \in V_{2}, v \in V_{3}} x^{d(u, v)}
\end{gathered}
$$




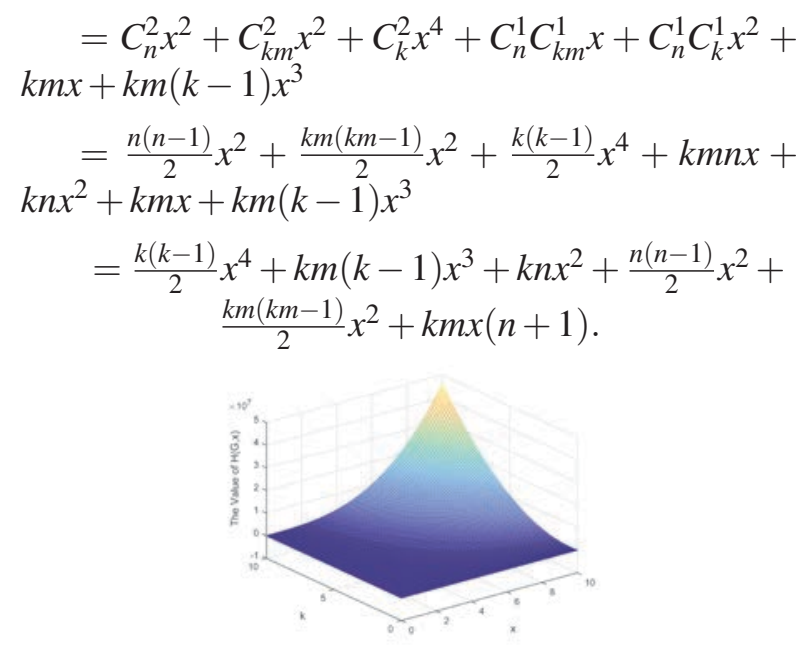

(a) $m=5, n=4$.

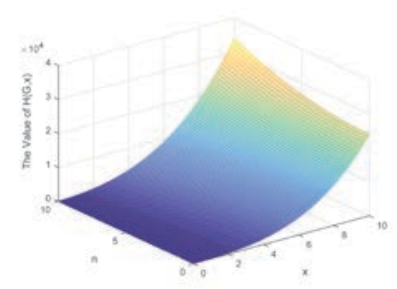

(b) $k=2, n=4$.

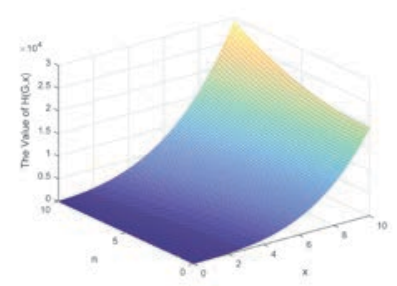

(c) $k=2, m=3$.

Figure 8. Computer-based comparative graph of the $H(G, x)$ index for $P N N(n, k, m)$ and its expression is $H(G, x)=\frac{k(k-1)}{2} x^{4}+k m(k-1) x^{3}+$ $k n x^{2}+\frac{n(n-1)}{2} x^{2}+\frac{k m(k m-1)}{2} x^{2}+k m x(n+1)$.

Theorem 5 Let $G \cong P N N(n, k, m)$, where $n, k, m \geq$ 1. Then, the first Zagreb index and second Zagreb index are as follows

$$
\begin{aligned}
& \overline{M_{1}}(G)=k m\left[n^{2}+(n+1)(k+k m-1)-1\right] . \\
& \overline{M_{2}}(G)=\frac{k^{2} m^{2} n(n+1)}{2}+\frac{k m(k m-1)(n+1)^{2}}{2}+\frac{k m^{2}(k-1)}{2} .
\end{aligned}
$$

Proof. Noting the definitions of $\overline{M_{1}}(G)$ and $\overline{M_{2}}(G)$, $V(G)$ have the following four cases for calculating $\overline{M_{1}}(G)$ and $\overline{M_{2}}(G)$. Moreover, based on the following cases, any two distinct vertices $u$ and $v$ are not adjacent:
(1) $u, v \in V_{1}$,
(2) $u, v \in V_{2}$,
(3) $u, v \in V_{3}$,
(4) $u \in V_{1}, v \in V_{3}$.

In $P N N(n, k, m)$, we firstly consider the $\overline{M_{1}}(G)=$ $\sum_{u \neq v, u v \notin E(G)}[d(u)+d(v)]$. Then, we have

$$
\begin{aligned}
\overline{M_{1}}(G) & \sum_{u, v \in V_{1}}[d(u)+d(v)]+\sum_{u, v \in V_{2}}[d(u)+d(v)] \\
& +\sum_{u, v \in V_{3}}[d(u)+d(v)]+\sum_{u \in V_{1}, v \in V_{3}}[d(u)+d(v)] \\
= & 2 k m C_{n}^{2}+2(n+1) C_{k m}^{2}+2 m C_{k}^{2}+C_{n}^{1} C_{k}^{1}(k m+m) \\
= & k m\left[n^{2}+(n+1)(k+k m-1)-1\right] .
\end{aligned}
$$

Similarly, according to the definition of $\overline{M_{2}}(G)=$ $\sum_{u \neq v, u v \notin E(G)}[d(u) \times d(v)]$, and its situations are same as $\overline{M_{1}}(G)$, then we can obtain

$$
\begin{aligned}
& \overline{M_{2}}(G)=\sum_{u, v \in V_{1}}[d(u) \times d(v)]+\sum_{u, v \in V_{2}}[d(u) \times \\
& d(v)]+\sum_{u, v \in V_{3}}[d(u) \times d(v)]+\sum_{u \in V_{1}, v \in V_{3}}[d(u) \times \\
& d(v)] \\
& \quad=C_{n}^{2} k m \cdot k m+C_{k m}^{2}(n+1)(n+1)+C_{k}^{2} m \cdot m+ \\
& C_{n}^{1} C_{k}^{1} k m \cdot m \\
& =k^{2} m^{2} \cdot \frac{n(n-1)}{2}+(n+1)^{2} \cdot \frac{k m(k m-1)}{2}+m^{2} . \\
& \frac{k(k-1)}{2}+k^{2} m^{2} n \\
& =\frac{k^{2} m^{2} n(n+1)}{2}+\frac{k m(k m-1)(n+1)^{2}}{2}+\frac{k m^{2}(k-1)}{2} .
\end{aligned}
$$$$
d(v)]
$$

Theorem 6 Let $G \cong P N N(n, k, m) n, k, m \geq 1$. Then, the expression of $\bar{F}(G)$ is given as

$$
\begin{aligned}
& \bar{F}(G)=k m^{2}\left(k n^{2}-k n+k-1\right)+k m(k m- \\
& 1)(n+1)^{2}+k m^{2} n\left(k^{2}+1\right) .
\end{aligned}
$$

Proof. According to the definition of $\bar{F}(G)$ in Section 2, there are the following four cases
(1) $u, v \in V_{1}$,
(2) $u, v \in V_{2}$,
(3) $u, v \in V_{3}$,
(4) $u \in V_{1}, v \in V_{3}$.

Based on the $\bar{F}(G)=\sum_{u v \notin E(G)}\left[d^{2}(u)+d^{2}(v)\right]$ and the above cases, one can get

$$
\bar{F}(G)=\sum_{u, v \in V_{1}}\left[d^{2}(u)+d^{2}(v)\right]+\sum_{u, v \in V_{2}}\left[d^{2}(u)+\right.
$$
$\left.d^{2}(v)\right]+\sum_{u, v \in V_{3}}\left[d^{2}(u)+d^{2}(v)\right]+\sum_{u \in V_{1}, v \in V_{3}}\left[d^{2}(u)+\right.$ $\left.d^{2}(v)\right]$ 


$$
\begin{aligned}
& \quad=2 k^{2} m^{2} C_{n}^{2}+2(n+1)^{2} C_{k m}^{2}+2 m^{2} C_{k}^{2}+ \\
& k n\left[(k m)^{2}+m^{2}\right] \\
& \quad=n(n-1) k^{2} m^{2}+k m(k m-1)(n+1)^{2}+k(k- \\
& \text { 1) } m^{2}+n k\left[m^{2}\left(k^{2}+1\right)\right] \\
& \quad=k m^{2}\left(k n^{2}-k n+k-1\right)+k m(k m-1)(n+1)^{2}+ \\
& k m^{2} n\left(k^{2}+1\right) .
\end{aligned}
$$

Theorem 7 Let $G \cong P N N(n, k, m)$, where $n, k, m \geq$ 1. Then the expression of $\operatorname{irr}(G)$ is given as

$$
\operatorname{irr}(G)=n k m|k m-n-1|+m k|n+1-m| .
$$

Proof. According to the definition of $\operatorname{irr}(G)$, just only two cases are known

$$
\text { (1) } u \in V_{1}, v \in V_{2},(2) u \in V_{2}, v \in V_{3} .
$$

Based on the above cases and the $\operatorname{irr}(G)=$ $\sum_{u v \in E(G)}|d(u)-d(v)|$, we can arrive at

$$
\begin{aligned}
& \quad \operatorname{irr}(G)=\sum_{u v \subseteq E(G)}\left|d\left(V_{1}\right)-d\left(V_{2}\right)\right|+\sum_{u v \subseteq E(G)} \mid \\
& d\left(V_{2}\right)-d\left(V_{3}\right) \mid \\
& \quad=C_{n}^{1} C_{k m}^{1}|k m-n-1|+C_{k m} C_{k}^{1}|n+1-m| \\
& \quad=n k m|k m-n-1|+k^{2} m|n+1-m| .
\end{aligned}
$$

Theorem 8 Let $G \cong P N N(n, k, m)$, where $n, k, m \geq$ 1. Then, the first multiplication Zagreb index is given as

$$
\prod_{1}(G)=(k m)^{2 n}(n+1)^{2 k m} m^{2 k} .
$$

Theorem 9 Let $G \cong P N N(n, k, m)$, where $n, k, m \geq$ 1. Then the expression of $N K(G)$ is given as

$$
N K(G)=(k m)^{n}(n+1)^{k m} m^{k} .
$$

Theorem 10 Let $G \cong \operatorname{PNN}(n, k, m)$, where $n, k, m \geq 1$. Then the reciprocal complementary Wiener index is given as $\frac{k(k-1)}{2}$.

$R C W(G)=\frac{n(2 k+n-1)}{6}+\frac{k m(2 k m+3 n+6 k-5)}{12}+$

Proof. Based on the definition of $R C W(G)$ in Section 2 , there are the following six cases
(1) $u, v \in V_{1}$,
(2) $u, v \in V_{2}$,
(3) $u, v \in V_{3}$,
(4) $u \in V_{1}, v \in V_{2}$,
(5) $u \in V_{1}, v \in V_{3}$,
(6) $u \in V_{2}, v \in V_{3}$.

According to the above cases and $R C W(G)=$ $\sum_{\{u, v\} \subseteq V(G) \frac{1}{d+1-d(u, v)}}$, we have

$$
\begin{gathered}
R C W(G)=\sum_{u, v \in V_{1}} \frac{1}{d+1-d(u, v)}+\sum_{u, v \in V_{2}} \frac{1}{d+1-d(u, v)}+ \\
\sum_{u, v \in V_{3}} \frac{1}{d+1-d(u, v)}+\sum_{u \in V_{1}, v \in V_{2} \frac{1}{d+1-d(u, v)}+}+ \\
\sum_{u \in V_{1}, v \in V_{3}} \frac{1}{d+1-d(u, v)}+\sum_{u \in V_{2}, v \in V_{3} \frac{1}{d+1-d(u, v)}}
\end{gathered}
$$

$=\frac{1}{d+1-2} C_{n}^{2}+\frac{1}{d+1-2} C_{k m}^{2}+\frac{1}{d+1-4} C_{k}^{2}+$
$\frac{1}{d+1-1} k m n+\frac{1}{d+1-2} k n+\frac{1}{d+1-1} k m+\frac{1}{d+1-3} k m(k-1)$
$\quad=\frac{n(n-1)}{6}+\frac{k m(k m-1)}{6}+\frac{k(k-1)}{2}+\frac{k m n}{4}+\frac{k n}{3}+\frac{k m}{4}+$
$\frac{k m(k-1)}{2}$

$$
=\frac{n(2 k+n-1)}{6}+\frac{k m(2 k m+3 n+6 k-5)}{12}+\frac{k(k-1)}{2} .
$$

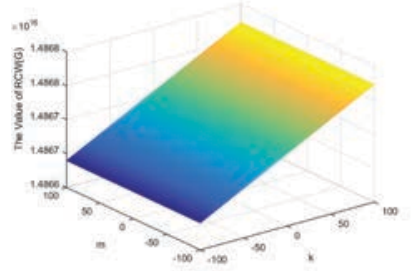

(a) $n=100$.

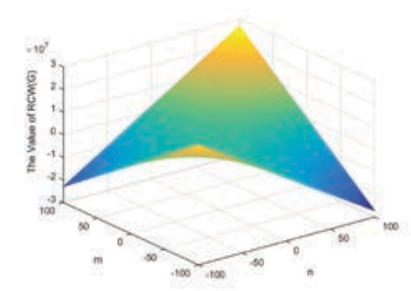

(b) $k=5$.

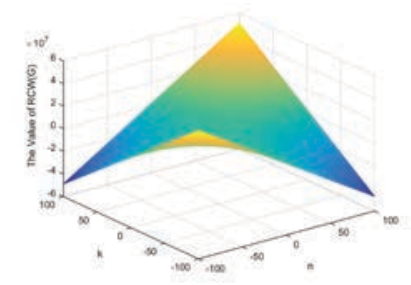

(c) $m=10$.

Figure 10. Computer-based comparative graph of the $R C W(G)$ index for $P N N(n, k, m)$ and its expression is

$R C W(G)=\frac{n(2 k+n-1)}{6}+\frac{k m(2 k m+3 n+6 k-5)}{12}+\frac{k(k-1)}{2}$.

\section{Conclusion}

In this paper, we calculated the degree-based topological indices (TI's) as well as distancedbased TI's of $\operatorname{PNN}(n, k, m)$. Furthermore, the analytical closed formulas of degree-based and 
distance-based indices for the network were manifested, which will help the scholars to understand and explore the underlying topologies of $\operatorname{PNN}(n, k, m)$, which are working in network science and physical features. In order to help us to know the properties of topological indices, which we had already computed, we plotted the three-dimensional graphics of the $W(G), H(G)$, $H_{t}(G), H_{A}(G), H_{M}(G), D D(G), H(G, x), N K(G)$ and $R C W(G)$ with the help of space cartesian coordinate system.

\section{Acknowledgement}

The work of was partly supported by the National Science Foundation of China under Grant no. 11601006, and China Postdoctoral Science Foundation under Grant no. 2017M621579, the Postdoctoral Science Foundation of Jiangsu Province under Grant no. 1701081B, the Natural Science Foundation for the Higher Education Institutions of Anhui Province of China under Grant no. KJ2015A331.

\section{Conflict of Interest}

The authors declare that they have no conflict of interest.

\section{References}

[1] J. Cao, R. Li, Fixed-time synchronization of delayed memristor-based recurrent neural networks, Sci. China. Inf. Sci. 60(3) (2017) 032201.

[2] Y. Huo, J. B-Liu, J. Cao, Synchronization analysis of coupled calcium oscillators based on two regular coupling schemes, Neurocomputing 165 (2015) 126-132.

[3] Z. Guo, J. Wang, Z. Yan, Attractivity analysis of memristor-based cellular neural networks with time-varying delays, IEEE Trans. Neural Netw. Learn. Syst. 25 (2013) 704-717.

[4] J. Devillers, A. T. Balaban, Topological Indices and Related Descriptors in QSAR and QSPR, Gordon Breach, Amsterdam 1999.

[5] M. Karelson, Molecular Descriptors in QSAR/QSPR, Wiley, New York, 2000.

[6] M. Javaid, J. B-Liu, M. A. Rehman, S. H. Wang, On the Certain Topological Indices of Titania Nan- otube $\mathrm{TiO}_{2}[m, n]$, Zeitschrift für Naturforschung A 72(7) 2017 647-654.

[7] M. Imran, S. Hafi, W. Gao, M. R. Farahani, On topological properties of sierpinski networks, Chaos, Solitons and Fractals 98 (2017) 199-204.

[8] S. H. Wang, B. Wei, Multiplicative Zagreb indices of $k$-trees, Discrete Appl. Math. 180 (2015) 168175.

[9] M. Javaid, Jinde Cao, Computing topological indices of probabilistic neural network, Neural Comput. Applic. (2017). doi:10.1007/s 00521-0172972.

[10] W. Gao, M. K. Siddiqui, Molecular descriptors of nanotube, oxide, silicate, and triangulene networks, Journal of Chemistry 2017 (2017).

[11] J. B-Liu, S. H. Wang, C. Wang, S. Haya, Further results on computation of topological indices of certain networks, IET Control Theory \& Applications 11(13) (2017) 2065-2071.

[12] J. A. Bondy, U. S. R. Murty, Graph Theory with Applications, Macmillan, New York, 1976.

[13] H. Wiener, Structural determination of the paraffin boiling points, J. Am. Chem. Soc. 69 (1947) 17-20.

[14] O. Ivanciuc, T. S. Balaban, A. T. Balaban, Reciprocal distance matrix, related local vertex invariants and topological indices, J. Math. Chem. 12 (1993) 309-318.

[15] R. Todeschini, V. Consonni, Molecular descriptors for chemoinformatics, vol I, vol II. Wiley-VCH, Weinheim (2009) 934-938.

[16] K. Xu, K. C. Das, N. Trinajstic, The Harary Index of a Graph, Springer Briefs in Mathematical Methods, DOI:10.1007/97836624584335.

[17] I. Gutman, Selected properties of the Schultz molecular topological index, J. Chem. Inf. Comput. Sci. 34 (1994) 1087-1089.

[18] H. Narumi, M. Hatayama, Simple topological index. a newly devised index charaterizing the topological nature of structural isomers of saturated hydrocarbons, Mem. Fac. Eng. Hokkaido Univ. 16 (1984) 209-214.

[19] D. J. Klein, V. R. Rosenfeld, The NarumiKatayama degree-product index and the degreeproduct polynomial, in: I. Gutman, B. Furtula (Eds.), Novel Molecular Structure DescriptorsTheory and Applications II, Univ. Kragujevac, Kragujevac (2010) 79-90.

[20] I. Gutman, Some Properties of the Wiener Polynomials, Graph Theory Notes New York 25 (1993) 13-18. 
[21] C. Wang, J.-B.Liu, S. Wang, Sharp upper bounds for multiplicative Zagreb indices of bipartite graphs with given diameter, Discrete Appl. Math. 227 (2017) 156-165.

[22] I. Gutman, N. Trinajstić, Graph theory and molecular orbits. Total $\pi$-electron energy of alternant hydrocarbons, Chem. Phys. Lett. 17 (1972) 535-538.

[23] B. Furtula, I. Gutman, Z. Kovijanić Vukićević, G. Lekishvili, G. Popivoda, On an old/new degreebased topological index, Sciences mathematiques 40 (2015) 19-31.

[24] S. Mukwembi, A note on diameter and the degree sequence of a graph, Appl. Math. Lett. 25 (2012) 175-178.

[25] R. Todeschini, D. Ballabio, V. Consonni, Novel molecular descriptors based on functions of new vertex degrees, in: I. Gutman, B. Furtula (Eds.), Novel Molecular Structure Descriptors Theory and Applications I, Univ. Kragujevac, Kragujevac (2010) 72-100.

[26] R. Todeschini, V. Consonni, New local vertex invariants and molecular descriptors based on functions of the vertex degrees, MATCH Commun. Math. Comput. Chem. 64 (2010) 359-372.

[27] S. Wang, B. Wei, Multiplicative Zagreb indices of k-trees, Discrete Appl. Math. 180 (2015) 168-175.

[28] O. Ivanciuc, QSAR comparative study of Wiener descriptors for weighted molecular graphs, J. Chem. Inf. Comput. Sci. 40 (2000) 1412-1422.

[29] O. Ivanciuc, T. Ivanciuc, AT. Balaban, The complementary distance matrix, a new molecular graph metric, ACH Models Chem. 137 (2000) 57-82.

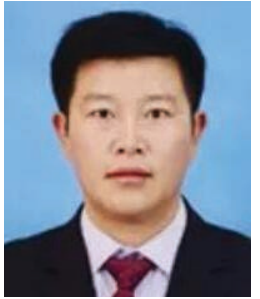

Jia-Bao Liu is currently an Associate Professor at School of Mathematics and Physics, Anhui Jianzhu University, Hefei 230601, China. He received the B.S. degree in Mathematics and Applied Mathematics from Wanxi University, Anhui, China, in 2005, and the M.S. degree and Ph.D. degree in Mathematics and Applied Mathematics from Anhui University, Anhui, China, in 2009 and 2016, respectively. From September 2013 to July 2014, he was a Visiting Researcher at the School of Mathematics, Southeast University, China. In March 2017, he was a Postdoctoral Fellow at the School of Mathematics, Southeast University, China. His current research interests include graph theory and its applications, fractional calculus theory, neural networks, and complex dynamical networks. Dr. Liu is a Reviewer of Mathematical Reviews and Zentralblatt-Math.

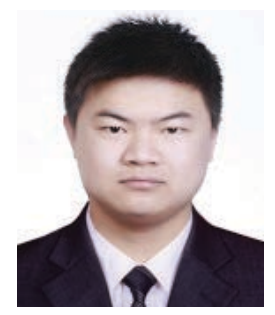

Jing Zhao is a master student at Anhui Jianzhu University, majoring in system engineering. He received the B.S. degree from Tongling University, Anhui, China, in 2017. His current research interests include graph theory and its applications, fractional calculus theory, neural networks, and complex dynamical networks.

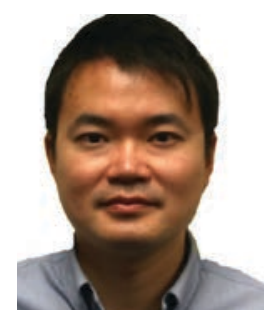

Shaohui Wang is an assistant professor at Savannah State University since August 2017. His research focused on Discrete Mathematics, including Graph Theory and Combinatorics. He was working in Adelphi University, NY from 2016-2017. He received the Ph.D. of Mathematics with a minor in Graph Theory at the University of Mississippi in August 2016. He also obtained the B.S. degree with a major of Mathematics and Applied Mathematics at Hubei
University of Education in June 2009, the first M.S. degree with a major of Operations Research and Cybernetics at Central China Normal University in June 2012, the second M.S. degree with a major of Mathematics with a minor in Statistics at the University of Mississippi in December 2014. He has published many papers in various journal. Furthermore, he is serving Associate Editors in two journals: Journal of Undergraduate Mathematics and Its Applications and SCIREA Journal of Mathematics; and a reviewer of over 20 mathematical journals.

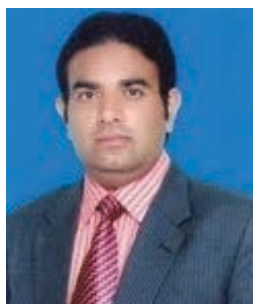

M. Javaid completed his Post Doctorate Mathematics (2015-17) from School of Mathematical Sciences, University of Science and Technology of China, Hefei, China and Ph.D. Mathematics (2009-14) from National University of Computer and Emerging Sciences, Lahore, Pakistan. He is currently working in different areas of graph theory such as spectral theory of graphs, computational graph theory and chemical graph theory.

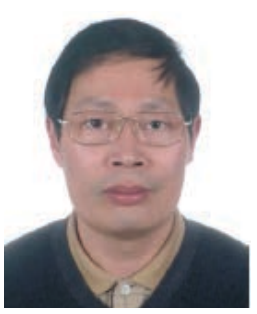

Jinde Cao is an Endowed Chair Professor, the Dean of School of Mathematics and the Director of the Research Center for Complex Systems and Network Sciences at Southeast University. From March 1989 to May 2000, he was with the Yunnan University. In May 2000, he joined the School of Mathematics, Southeast University, Nanjing, China. From July 2001 to June 2002, he was a Postdoctoral Research Fellow at Chinese University of Hong Kong, Hong Kong. Professor Cao was an Associate Editor of the IEEE Transactions on Neural Networks, and Neurocomputing. He is an Associate Editor of the IEEE Transactions on Cybernetics, IEEE Transactions on Cognitive and Developmental Systems, Journal of the Franklin Institute, Mathematics and Computers in Simulation, Cognitive Neurodynamics, and Neural Ne- 
tworks. He is a Fellow of IEEE, a Member of the Academy of Europe, a Member of European Academy of Sciences and Arts, and a Foreign Fellow of Pakistan Academy of Sciences. He has been named as Highly-Cited Researcher in Engineering, Computer Science, and Mathematics by Thomson Reuters/Clarivate Analytics. He received the National Innovation Award of China (2017). 